\title{
Patterns and Temporal Trends of 7 Major Organic Solvent Induced Occupational Diseases in Guangdong, China 2006-2015
}

\author{
Xudong Li \\ Department of Epidemiology, School of Public Health, Southern Medical University \\ Hongwei Tu \\ Guangdong Provincial Center for Disease Control and Prevention \\ Shijie Hu \\ Guangdong Province Hospital for Occupational Disease Prevention and Treatment \\ Jiabing Chen \\ Guangdong Province Hospital for Occupational Disease Prevention and Treatment \\ Hongwei Yu \\ Guangdong Province Hospital for Occupational Disease Prevention and Treatment \\ Runze Zhang \\ Department of Epidemiology, School of Public Health, Southern Medical University \\ Peiliang Chen \\ Department of Epidemiology, School of Public Health, Southern Medical University \\ Hongying Qu ( $\nabla$ gdzfyb@126.com ) \\ Guangdong Province Hospital for Occupational Disease Prevention and Treatment \\ Qing Chen \\ Department of Epidemiology, School of Public Health, Southern Medical University
}

\section{Research article}

Keywords: Organic solvent, Occupational diseases, Patterns, Trends, Guangdong

Posted Date: September 7th, 2019

DOI: https://doi.org/10.21203/rs.2.14039/v1

License: (9) (i) This work is licensed under a Creative Commons Attribution 4.0 International License. Read Full License 


\section{Abstract}

(1)Background: This study aimed to describe the distribution characteristics and temporal trends of major Organic Solvent induced occupational diseases (OSODs) in Guangdong Province, China.

(2) Methods: A total of 1320 OSODs cases diagnosed from 2006 to 2015 were involved in this study, including information of case distribution, working age, industrial type, economic type and enterprise scale. 7 major OSODs accounted for the majority cases (1290 cases, $98 \%$ ), including benzolism, n-hexane poisoning, 1,2-dichloroethane poisoning, trichloromethane poisoning, dimethyl formamide poisoning, benzene-induced leukemia and trichloroethyleneinduced medicamentosa-like dermatitis.

(3) Results: The incidence of OSODs was relatively higher in Shenzhen, Dongguan and Guangzhou than other districts. Most cases were related to light industry and electronics for industry type and small and medium-sized and private enterprises for enterprise scales and ownership types. Notably, the case number of benzolism and benzene-induced leukemia presented an increasing trend with year. The onset age of benzolism exhibited an increasing trend with year. The working age of onset of 1,2-dichloroethane poisoning, trichloromethane poisoning and dimethyl formamide poisoning were shorter than others.

(4) Conclusions: Since the control and prevention of 7 major OSODs has achieved remarkable results, more measurements are needed to control benzolism and benzene-induced leukemia cases in Guangdong Province.

\section{Introduction}

Organic solvents are a group of diverse chemical compounds widely used in industry due to properties convenient for extracting, dissolving, or diluting materials. Their inclusion in numerous products such as paints, degreasers, glues, inks, pharmaceuticals, pesticides, and cosmetic and cleaning products results in exposures in diverse occupational settings[1]. Occupational exposure to organic solvents and the resulting health damages that is Organic solvent induced occupational disease (OSOD) have raised concern[2]. In China, There were 58 severe acute occupational poisoning accidents related to organic solvents from 1989 to 2003 with 393workers poisoned and 48 workers died [3]. Guangdong Province of China, covering an area of 179,700 m², has a total population of 108 million and a working-age population of over 80 million. It is a world-renowned manufacturing center for producing bags, suitcases, footwear, electronic components and chemical industry, so that a large number of people could have occupational exposure to organic solvents. .Since the 1990 s, about $40 \%$ of the workers could have occupational exposure to organic solvents[4]. OSODs had accounted for more than $70 \%$ of occupational poisoning cases in Guangdong Province[5, 6].

Notably, accumulating evidence has drawn attention to OSOD as stated above. However, to the best of our knowledge, there was no previous study arising from large samples over a long period of time on patterns and temporal trends of OSOD in Guangdong. Therefore, we initially performed this study using National Occupational Disease and Occupational Health Information Monitoring System (NODOHIMS) [7]. to explore the patterns and temporal trends of major OSODs in Guangdong during 2006-2015. Our study not only provided valuable information for the control and prevention of OSODs in Guangdong Province, but also provided reference for the prevention and control of occupational Organic solvents hazards in developing countries in the stage of rapid industrialization.

\section{Materials And Methods}

\subsection{Data collection}

Information of OSOD cases during 2006-2015 in Guangdong Province was audited from the NODOHIMS. The occupational disease cases were ascertained by state-authorized diagnosis institutes for occupational diseases, gathered through the hierarchies of occupational health authorities in the reporting system, and finally compiled by Chinese Centre for Disease Control and Prevention (CDC). The occupational disease report cards were examined by the relevant health administrative departments at the county, city and provincial levels. Taking the diagnostic time reported in the Occupational Disease Report Card as the boundary point, case admission and deadline points were January 1, 2006 and December 31, 2015, respectively. The study was approved by the ethics committee of Guangdong Province Hospital for Occupational Disease Prevention and Treatment.The analyses of the development of occupational diseases included changes in case numbers, the distribution of cases between industrial sectors, vulnerable work populations, enterprises with high risks, and major hazardous factors.

Occupational diseases were classified on basis of the List of Occupation Diseases of China. Industries were classified according to the International Standard Industrial Classification (ISIC Rev4.0囚and Industrial Classification for National Economic Activities (GB/T4754-2011). Enterprise scale were classified according to the Regulations of Classification of small-sized, medium-sized and large-sized enterprises on statistics (Tentative) (GTZ [2017] No. 17): 1) large-sized enterprises: numbers of employees are more than 1000 and operating income is greater than 400 million RMB; 2) medium-sized enterprises: numbers of employees are more than 300 but less than 1000, and operating income is greater than 20 but less than 400 million RMB; 3 ) small enterprises: numbers of employees are more than 20 but less than 300, and operating income is greater than 3 but less than 20 million RMB; 4 ) microenterprises: numbers of employees people are less than 20, or operating income is less than 3 million RMB. According to Provisions on the Classification of Business Registration Types, economic type of enterprises were divided into "Foreign Funded Economy”, " Private Economy”, "Collective Economy”, "Stateowned Economy”, "Hong Kong/Macao/Taiwan Economy” respectively.

\subsection{Diagnostic of OSODs}




\subsubsection{Benzolism}

According to the Chinese Diagnostic Criteria [8],the occurrence of benzolism was identified based on having a long-term occupational exposure histroy to benzene, with clinical manifestations of hematopoietic system damage; a) the white blood cell count lower than $4 \times 10^{9} / L$ or the number of neutrophils less than $2 \times 10^{9} / \mathrm{L} ;$ b) platelet count less than $80 \times 10^{9} / \mathrm{L}$.

\subsubsection{Benzene-induced leukemia}

According to the Chinese Diagnostic Criteria [9],the occurrence of Benzene-induced leukemia was identified based on suffering from chronic occupational benzene poisoning or having the medical history of occupational chronic benzene toxonosis getting the leukemia. Any patient without the medical history of occupational chronic benzolism should be so diagnosed based on satisfaction of all the three following conditions: positive diagnosis of leukemia, positive occupational history containing exposure to benzene, and engagement of any job exposed to benzene for 6 months (included) or more accumulatively, experiencing a prepatent period of 2 years (included) or more.

\subsubsection{N-hexane poisoning}

According to the Chinese Diagnostic Criteria [10],the occurrence of N-hexane poisoning was identified based on an long-term occupational exposure history to normal hexane shows the clinical manifestations mainly by multiple peripheral nerve damage and one of following items: a) The distal parts of extremities feel symmetrically distributed pain, hypesthesia or disturbance of tuning fork vibration sensing accompanying with Achilles tendon hyporeflexia; b) the nerve-electromyography shows positive neurogenic damage.

\subsubsection{Trichloroethylene-induced medicamentosa-like dermatitis}

According to the Chinese Diagnostic Criteria [11],the occurrence of Trichloroethylene-induced medicamentosa-like dermatitis was identified based on an occupational exposure history to trichlorethylene; skin lesion is acute dermatitis, mostly commonly appears as exfoliative dermatitis, some appears as erythema multiforme, Stevens-Johnson syndrome or epidermolysis bullosa; frequently with fever, liver damage and superficial lymph node enlargement.

\subsubsection{1,2-dichloroethane poisoning}

According to the Chinese Diagnostic Criteria [12],the occurrence of 1,2-dichloroethane poisoning was identified based on an short-term occupational exposure history to dichloroethane of high concentration shows the clinical manifestations mainly by central nervous system damage and such central nervous system symptoms as dizziness, headache and debilitation, who may also feel nausea, emesis or irritation of eyes or the upper respiratory tract.

\subsubsection{Trichloromethane poisoning}

According to the Chinese Diagnostic Criteria[13], the occurrence of Trichloromethane poisoning was identified based on an occupational history positively related to the poisoning, including the specific type of the occupational toxicant, the exposure mode and the reason for intensive absorption in a short period shows corresponding clinical manifestations of acute liver damage, excluding similar diseases caused by other reasons.

\subsubsection{Dimethyl Formamide Poisoning}

According to the Chinese Diagnostic Criteria [14],the occurrence of Dimethyl Formamide Poisoning was identified based on A short-term exposure to dimethylamide resulted in dizziness, nausea, vomiting, loss of appetite, abdominal pain and acute toxic liver disease.

\subsection{Statistical analyses}

A descriptive analysis was performed to explore the characteristics of the OSODs reported in Guangdong during 2006-2015, including annual changes of reported occupational disease cases, distribution of disease categories, regional distribution, average working age of onset, and the overall annual changing trend of age of onset. Moreover, a linear trend test was conducted for number of cases, median working age of onset, and median for age of onset with curve fit method, taking the diagnosis year as the independent variable. A trend test was conducted by the chi-square test for distribution of major OSODs in industrial type, ownership type and enterprise scale. All the data cleaning and analysis were conducted using SPSS20.0 software (SPSS Inc., Chicago, IL, USA).

\section{Results}

\subsection{Changes in the Number of OSODs Cases Reported from 2006-2015}


Of reported 1320 cases of OSODs during 2006-2015,1290 cases (98\%) were from the 7 major OSODs, including benzolism (592 cases), benzene-induced leukemia (232 cases), n-hexane poisoning (206 cases), trichloroethylene-induced medicamentosa-like dermatitis ( 94 cases), 1,2-dichloroethane poisoning (65 cases), trichloromethane poisoning (52 cases), and dimethyl formamide poisoning (49 cases).

Annual trends of the 7 major OSODs in Guangdong are shown in Table 1.The minimum case number of OSODs among these years was 65 in 2007 , while the maximum one is 205 in 2012. annual trends of the case numbers of the 7 major OSODs did not show statistics significance.The minimum case number of benzolism among these years was 26 in 2008, while the maximum one is 121 in 2014 . The minimum case number of benzene-induced leukemia was 2 in 2006, while the maximum one was 43 in 2011. The fitted trend curve of benzolism and benzene-induced leukemia during 2006-2015 exhibited an exponential increase of new cases $\left(R^{2}=0.663, R^{2}=0.706, P<0.01\right)$. However, annual trends of the case numbers of other OSODs did not show statistics significance.

\subsection{Regional and Gender Distribution of OSODs Cases Reported from 2006-2015}

The top three cities reporting the most cases of OSODs were Shenzhen(468cases, 36.3\%),Dongguan(411cases, $31.9 \%)$ and Guanghzou (167cases, $12.9 \%$ ). Heyuan, Meizhou,Shantou,Shanwei, Shaoguan, Yangjiang, Yunfu had no OSODs cases reported. Benzolism and Benzene-induced leukemia are mainly distributed in three cities of Guangdong, Shenzhen and Dongguan. But Benzolism cases are mainly distributed in Shenzhen (217 cases, $36.7 \%$ ) while Benzene-induced leukemia cases are more common in Dongguan(100 cases, $43.1 \%$ ).

There were slightly more males than females in gender(699 cases vs 591 cases). but less males than females in $n$-hexane poisoning(159 cases vs 47 cases)

Figure 1 and Figure 2 show more details.

\subsection{Industry, Enterprise Ownership Type and Scale Distribution of OSODs Reported from 2006-2015}

Most of the OSODs cases were distributed in light industry (260 cases, 43.9\%) and electron industry (46 cases, $48.9 \%)$. In addition to Trichloromethane poisoning and Trichloroethylene-induced medicamentosa-like dermatitis,which are mainly distributed in the electronics industry, other OSODs mainly distributed in light industry.

Most of the OSODs cases were reported in private owend enterprise (638 cases, 49.5\%) and Hong Kong/ Macao/Taiwan owend enterprise (319cases, $24.7 \%$ ), while Benzolism cases are mainly distributed in foreign funded economy (200 cases, 33.8\%).

In the terms of the enterprise scale】a larger proportion of OSODs cases were distributed in small-sized ( 220 cases, $37.2 \%)$ and medium-sized enterprises (172 cases, 29.1\%). But Trichloromethane poisoning cases are mainly distributed in large-sized enterprises (30 cases, $57.7 \%)$.

Table 2 shows more details.

\subsection{Annual Trend of Onset Age}

Onset age of OSODs was young, while the median onset ages of trichloromethane poisoning was the youngest one with 21 years old and the median onset age of benzolism was theoldest one with 37 years old. The median onset age of benzolism increased from 31 in 2007 to 42 in 2015 . The median onset age of dimethyl formamide poisoning in 2006 was 32.5 years old, and that in 2015 was 51 years old. The fitted trend curve of benzolism onset age during $2006-$ 2015 presented an increasing trend in general and indicated liner relationship with year $\left(R^{2}=0.846, P<0.05\right)$. Onset age of dimethyl formamide poisoning also exhibited an increasing trend in general and showed liner relationship with year $\left(R^{2}=0.793, P<0.05\right)$. However, the annual trends of onset age of other OSODs did not show statistical significance. (Table 3)

\subsection{Annual Trend of Working Age of Onset}

The working age of onset of OSODs was generally short. The average working age of onset of trichloromethane poisoning, dimethyl formamide poisoning and occupational trichloroethylene-induced medicamentosa-like dermatitis was 1 month. Those of dichloroethane poisoning and $\mathrm{N}$-hexane poisoning were 5 and 8 months, respectively. The average exposure period at onset of benzolism and benzene-induced leukemia was 4 years. Time trends of exposure period did not show statistical significance (Table 4).

\section{Discussion}

The recognition that organic Solvent exhibits Occupational Diseases accelerator property has motivated growing interest in the research area of Occupational Health. This study aimed to explore the distribution characteristics and temporal trends of major OSODs in Guangdong during $2006-2015$.

In our study, the case numbers of benzolism and leukemia induced by benzene exhibited an exponential growth during 2006-2015 in Guangdong. The reasons of the rapid growth are as follows: Firstly, Guangdong is the one of provinces with huge demands for benzene production and usage, and thus have 
many occupational exposure groups. Some studies have indicated that the production and usage of pure benzene in China were increasing year by year. The average annual production and consumption capacity of pure benzene in China was increasing at the rates of $9.82 \%$ and $7 \%$, respectively[15]. Secondly, compared with the permissible concentration-time weighted average of $1 \mathrm{ppm}$ in major developed countries and regions, occupational exposure limit to benzene(that is $1.7 \mathrm{ppm}$ ) is still high in our country. A recent study has shown that the exposure concentration to benzene in China dropped from $215 \mathrm{mg} / \mathrm{m}^{3}$ during 1956-1978 to $82 \mathrm{mg} / \mathrm{m}^{3}$ during 1979-1989, and to $31 \mathrm{mg} / \mathrm{m}^{3}$ during 1990-2001 and then to $6 \mathrm{mg} / \mathrm{m}^{3}$ (1.7 ppm) during 2002-2005[16]. Another recent study has shown that exposure to benzene air $1 \mathrm{ppm}$ or even lower may cause hematotoxicity to susceptible populations[17]. Thirdly, nowadays the occupation involved with the use of adhesives and diluent containing benzene impurities is numerous. According to a study of Zhu et al [18], there are 26 benzene-containing solvents in 898 organic solvents, accounting for $2.9 \%$ of them. Such exposure to benzene lead to larger difficulties for benzene prevention and control. Ultimately, numerous measures have objectively decreased the rate of missed diagnosis and report of occupational poisoning and leukemia induced by benzene, such as the implementation of the responsibility of enterprises, the promoted supervision by regulatory authorities, the enhancement of laborers' right-protection awareness, the improvement of occupational physical examination standards, populations and exam rates, the elimination of barriers for accepting occupational disease diagnosis and the improvement of occupational disease report ability.

At same time, compared with occupational diseases induced by other organic solvents, the workers, enterprises, regulatory authorities and society have higher awareness and attention on benzolism and benzene-induced leukemia. More laborers may seek further examination and diagnosis in case of similar health damage and the technical service divisions have a higher ability and level to test the benzene and the occupational health examination. Our study showed that the reported benzolism age was increasing. Yet on the other hand, the prevention and control for benzolism in Guangdong has achieved some effects. In general, the protection for traditional occupational diseases in large enterprises should be better than that in small and medium enterprises. In our research, the reports of benzolism demonstrated an increasing trend with the rising proportion of large enterprises. This also indicated that the increase of reported cases of benzolism in Guangdong was not attributable to the poor prevention and control, but the rights and interests of laborers with benzolism were more extensively maintained.

Chronic N-hexane poisoning is caused by occupational poisoning and some of which are poisoned for long-time addicted to N-hexane. Our study suggested that the average working age for onset of $\mathrm{N}$-hexane poisoning was 8 months and the longest was 56 months. These data are consistent with the conclusions of the preliminary research on chronic N-hexane poisoning made by our hospital, in which the working year range was $3 \sim 39$ months and the average working year range was $(10.6 \pm 6.0)$ months [19]. These data are basically consistent with the conclusions of other domestic research [20]. It is reported in Chinese research that in the working place where the poisoning accidents occurred in the early 1990s, the N-hexane concentration in air was even up to $6000 \sim 9000 \mathrm{mg} / \mathrm{m}^{3}$. With the introduction of relevant national laws, standards and management systems, as well as the raising safety and health awareness of enterprises and laborers, the N-hexane concentration in air of the working place was gradually declining. However, among the enterprises having poisoning accidents, the over-limit rate of $\mathrm{N}$-hexane concentration was still up to $96.7 \%$.

Hexane poisoning was mainly centralized in small and private enterprises in Shenzhen and Dongguan of Guangdong with statistical significance. Combining with the Research and relevant preliminary researches [21], we speculated that the reasons for occurrence of occupational N-hexane poisoning accidents included: N-hexane poisoning mostly occurred in labor-intensive enterprises (e.g. shoemaking, leather, suitcase \& bag enterprises), which had large mobility of worker that were still dominated by handwork and short of necessary occupational health monitoring, as well as had difficulties in conducting occupational health monitoring, so as to cause that the $\mathrm{N}$-hexane damage to health could not be found and treated as earlier as possible. The companies were not well aware of the harm of $\mathrm{N}$-hexane, and used a large amount of $\mathrm{N}$-hexane without taking targeted prevention and control measures as well as sufficient occupational disease protective measures, and proper ventilation and detoxification facilities were not provided, especially in winter business peak season, when the companies would conduct overtime work with doors and windows closed for a long time. The laborers also lacked protection awareness for occupational diseases, and the protective equipment are not effectively used.

The most cases of occupational trichloroethylene-induced medicamentosa-like dermatitis (TCE) in the world occur in Guangdong. There were 94 new cases in the whole province during 2006-2015, mainly concentrating in Shenzhen, Dongguan and Huizhou of Pearl River Delta area, with 79 cases taking up $80.9 \%$. As for types of economy, poisoning incidents mainly occurred in private economies, with 67 cases accounting for $71.3 \%$. As for enterprise scale, poisoning incidents increased in small-sized enterprises, with 43 cases accounting for $45.7 \%$. After the onset, the condition progressed rapidly; the complications were relatively numerous; the treatment was highly difficult and case-fatality rate was high [22]. Indicate that the pathogenesis should belong to allergic reaction, most probably type IV. However, since the industrial TCE that patients expose is complicated in components and mixed with stabilizer, further study shall be made to determine whether there are other elements in allergen besides TCE. The pathogenesis of occupational trichloroethyleneinduced medicamentosa-like dermatitis has not been clarified. Therefore, in order to strengthen the supervision management on relevant enterprises, enterprises shall be encouraged to adopt other new type of chemical reagents to replace trichloroethylene so as to prevent and control the occurrence of occupational trichloroethylene-induced medicamentosa-like dermatitis.

1,2-Dichloroethane (1,2-DCE) poisoning, Trichloromethane poisoning and Dimethyl Formamide poisoning are acute poisoning caused by short-term exposure to high concentrations. All three can be poisoned by the respiratory tract or skin. Our research shows that the working age of onset of these three were shorter than the other four major OSODs. Studies have indicated that 1, 2-DCE poisoning and Trichloromethane poisoning are cases where the working hours are too long (12-16h a day). Current researches have indicated that acute poisoning of these three have the following characteristics: 1 ) It occurs in the seriously over-standard working place. 2) The poisoning incidents mainly occur in rooms with poor mechanical ventilation. 3) Workers may work long hours for a consecutive time with insufficient protection.

1, 2-DCE poisoning is mainly manifested as systemic diseases, especially central nervous system damage. New cases of 1,2-DCE poisoning in Guangzhou mainly took place in 2009-2012, especially in 2012 when a poisoning incident occurred with 34 people poisoned. It was mainly caused by high

Page 5/11 
concentration 1,2-DCE in materials adopted by workers or in the air at working place.Minimum, maximum and average DCE concentration in workshops in which infected person work were $7.4 \mathrm{mg} / \mathrm{m} 3,1199 \mathrm{mg} / \mathrm{m} 3$ and $272.2 \mathrm{mg} / \mathrm{m} 3$, respectively. It is a remarkable fact that the $1,2-D C E$ incidents mainly occurred in the environment with similar 1,2-DCE concentration, but only a part of people were affected. It is considered that this may be related to accumulated concentration, individual sensitivity and individual protection effectiveness[23].

Trichloromethane is a protoplasmic toxicant, which can directly damage liver cells and its organelles (especially endoplasmic reticulum), and cause fatty degeneration and necrosis of liver cells. During 2006-2015, a total of 52 cases of trichloromethane poisoning occurred, while the main incidence occurred with 35 cases in 2009. Different from the other six main OSODs, Trichloroethane poisoning occurs mainly in large enterprises. The age of onset of trichloromethane is 21 years old and is the youngest of all 12 occupational diseases included in the study[24]. Trichloromethane poisoning was mainly caused by high concentration. The simulated operation test results showed that the time weighted average concentration of trichloromethane in the air of the patient's workplace in 8 hours was $602.1 \mathrm{mg} / \mathrm{m} 3,366.6-403.4 \mathrm{mg} / \mathrm{m3}$, which is 30 times and 17.3 19.2 times than that of occupational exposure limit[25].

The clinical manifestation of Dimethyl Formamide (DMF) poisoning is a systemic disease characterized by digestive system, especially liver damage. Studies have shown that there is a population susceptibility to liver damage caused by DMF poisoning. In recent years, acute DMF poisoning cases have occurred in garment processing which have used the same fabric to process garments. And the incidence rate is high. The incubation period of poisoning varies with the route of exposure and concentration. After inhalation of the respiratory tract, acute poisoning usually occurs after 6 to 24 hours[26]. The incubation period of skin contact is relatively long. Our research shows that from 2006 to 2015, 49 cases of new cases of DMF poisoning in Guangdong Province, mainly in 2009, 35 cases. The age of onset of DMF poisoning is 28 years old, and the individual age can reach 55 years old. Studies indicate that the age of onset shows an increasing trend. We would provide some suggestions on the prevention and control of OSODs: 1) Strengthening publicity and education on the occupational diseases caused by organic solvent and enhance the laborer and enterprises' awareness, belief and implementation of protections, especially for benzolism and benzene-induced leukemia 2) Further committing the main body obligation of enterprises and the regulatory responsibility of controlling authorities, and promote the processing measures (mainly referring to the substitute measures), engineering measures, personal protection measures, management measures and medical measures (i.e., occupational health examination) for protection of organic solvents in small and medium-sized private enterprises, so as to prevent occurrence of group occupational poisoning. It should be noted that protection should be also provided to exposed skins in addition to respiratory inhalation. 3) Executing monitoring on the organic solvent induced occupational diseases and evaluation of occupational health risk based on the actual situation of occupational diseases occurring in Guangdong, so as to know well about the high risk industries, high risk areas and high risk professions related to the organic solvent induced occupational diseases to finally realize real-time monitoring, accurate evaluation and effective response relevant risks and hazards. 4) Improving the technical level of technical organizations, especially scientifically coping with the new trend after release of new version of the Law on Prevention and Control of Occupational Diseases that occupational disease group diagnosis is not required and management of qualifications of occupational health examination organizations is not required either. 5) Enhancing scientific research on the organic solvent induced occupational diseases, focusing on the health effect of collective exposure to several organic solvents and to other occupational hazard factors, the health effect of new organic solvents and new poisonousness in known poisons.

\section{Conclusion}

Of reported 1320 cases of OSODs during 2006-2015,1290 cases (98\%) were from the 7 major OSODs, including benzolism (592 cases), n-hexane poisoning (206 cases), 1,2-dichloroethane poisoning (65 cases), trichloromethane poisoning (52 cases), dimethyl formamide poisoning (49 cases), benzene-induced leukemia (232 cases) and trichloroethylene-induced medicamentosa-like dermatitis (94 cases). Three of them are acute poisoning caused by exposure to high concentrations of poisons, including 1,2-dichloroethane poisoning, trichloromethane poisoning and dimethyl formamide poisoning. 1290 cases mainly occurred in Shenzhen, Dongguan and Guangzhou and these cases were mainly observed in light industry and electronics, small and medium-sized enterprises as well as private economy. Except Trichloromethane poisoning, which is more likely to occur in large enterprises. Among them, a higher rate was seen in males than that females. Benzene-induced leukemia and benzolism exhibited an increasing trend, while there was an increasing trend in the onset age of benzolism. In summary, the prevention and control of OSOD in Guangdong has achieved remarkable efforts, while benzolism and benzene-induced leukemia continues to be an important problem. This study may provide reference for the prevention and control of occupational hazards in developing countries in the stage of rapid industrialization.

\section{Declarations}

\section{Ethics approval and consent to participate}

This work was agreed by Medical Ethics Committee,Guangdong Province Hospital for Occupational Disease Prevention and Treatment.

Availability of data and materials

Not applicable.

Competing interests:

The authors declare no conflict of interest. The founding sponsors had no role in the design of the study; in the collection, analyses, or interpretation of data; in the writing of the manuscript, and in the decision to publish the results.

Funding: 
This work was supported by a Grant from Guangdong Province Hospital for Occupational Disease Prevention and Treatment $\varangle$ Guangdong Provincial Key Laboratory of Occupational Disease Prevention and Treatment (2017B030314152); and a Grant from Science and Technology Science Foundation of Guangdong (2011B050700001, 2013B021800176), and a Grant from Guangdong medical scientific research fund (A2015150هC2016014).

Author Contributions:

Xudong Li and Hongwei Tu analyzed data and Xudong Li wrote the paper. Hongying Qu Shijie Hu Jiabin Chen and Xianzhong Wen provided original data in Occupational Disease Hazard Factors Monitoring System and Hongwei Yu and Peiliang Chen processed the original data. Xudong Li and Runze Zhang critically revised the manuscript. Qing Chen designed main structure of this study and provided statistical support.

Acknowledgments:

We thank the doctors in Guangdong Prevention and Treatment Center for Occupational Diseases and Lab Associates for occupational diseases diagnosis. Data was supported by the Occupational Disease Hazard Factors Monitoring System of the China Disease Prevention and Control Information System.

\section{References}

1.Costet N, Béranger R, Garlantézec R, Rouget F, Monfort C, Cordier S, Pelé F, Chevrier C: Occupational exposure to organic solvents during pregnancy and childhood behavior: findings from the PELAGIE birth cohort (France, 2002-2013). Environmental Health 2018, 17(1):63.

2.Taofeeq O, Olayinka RG, Taiwo OO, Ganiyu AO, Kabiru DM, Suleiman MA, Oke O, Taiwo AO, Ganiyu DM, Kabiru MA: Organic Solvent Exposure: Hepatotoxicity, Nephrotoxicity, and Haematotoxicity Assessment amongst Vehicle Spray Painters in Ile-Ife, Nigeria. American Journal of Environmental Protection 2015, 3:95-99.

3.Zhang M, Li T, Wang HQ, Wang HF, Chen SY, Du XY, Qin J, Zhang S, Ji LY: Characterization of severe acute occupational poisoning accidents related to asphyxiating gases in Chin between 1989 and 2003. Chinese Journal of Industrial Hygiene \& Occupational Diseases 2006, 24(12):723.

4.Jia XD, Jin XP: Status and prevention of organic solvents occupational hazards in China. Chinese J Industrial Hygiene Occup Dis 2000, 18:65-67.

5.Wen XZ, Xu-Dong LI, Huang YS, Zheng QL: Analysis on occupational disease spectrum in Guangdong province,2006-2010. China Occupational Medicine 2014.

6.Hu Shijie, Jin Jiachun, Jiang Jiaxin, Yongshun. H: Characteristics of occupational poisoning in Guangdong Province, 2006-2010. South China J Prev Med 2012, 38(3):22-28.

7.Han L, Yao W, Bian Z, Zhao Y, Ni C: Characteristics and Trends of Pneumoconiosis in the Jiangsu Province, China, 2006-2017. International Journal of Environmental Research and Public Health 2019, 16(3):437.

8.Ministry of Health P.R China: GBZ 68-2008 Diagnostic Criteria of Occupational Benzene Poisoning. In.; 2008.

9.National Health and Family Planning Commission of PRC: GBZ 94-2014 Diagnostic Criteria of Occupational malignant tumor. 2014.

10.Ministry of Health P.R China: GBZ 84-2002 Diagnostic Criteria of Occupational Chronic n-Hexane Poisoning. In.; 2002.

11.Ministry of Health P.R China: GBZ 71-2002 Diagnostic Criteria of Occupational Acute Chemical Poisoning(General Rules). 2002.

12.Ministry of Health P.R China: GBZ 39-2002 Diagnostic Criteria of Occupational Acute 1,2-Dichloroethane Poisoning. In.; 2002.

13.Ministry of Health P.R China: GBZ 185-2006 Diagnostic Criteria of Occupational medicamentosa-like dermatitis due to trichloroethylene. In.; 2006.

14.National Health and Family Planning Commission of the PRC: GBZ 85-2014 Diagnostic criteria of occupational dimethyl formamide poisoning. 2014.

15.Zhaolin X, Ping S, Zhongbin Z, Xipeng J: Review and perspective of bene-induced health hazards. CHINESE JOURNAL OF INDUSTRIAL HYGIENE AND OCCUPATIONAL DISEASES 2005, 23(4):241-243.

16.Wong 0: Investigations of Benzene Exposure, Benzene Poisoning, and Malignancies in China. Regul Toxicol Pharmacol 2002, 35(1):126-135.

17.Qing L, Luoping Z, Guilan L, Roel V, Weinberg RS, Mustafa D, Rappaport SM, Min S, Alter BP, Yongji W: Hematotoxicity in workers exposed to low levels of benzene. Science 2004, 306(5702):1774-1776.

18.Zhiliang Z, Xiaorong X, Junhua W, Huizhen X, Xiaozuo X, Jiangwei Y: Analysis of main volatile chemical components of common organic solvents in recent five years. CHINESE JOURNAL OF INDUSTRIAL HYGIENE AND OCCUPATIONAL DISEASES 2010, 28(8):581-583.

19.Zhao QK, Zhou YL, Zhou ZJ: Review on Occupational Poisoning and Toxicology of N-hexane. Occupational Health \& Emergency Rescue 2009.

20.Puri V, Chaudhry N, M: N-hexane neuropathy in screen printers. Electromyography \& Clinical Neurophysiology 2007, 47(3):145.

21. Hunter D: The diseases of occupations, Sixth edition edn. London: Hodder and Stonghton; 1978.

Page $7 / 11$ 
22.Hormozi M, Ansari-Moghaddam A, Mirzaei R, Dehghan Haghighi J, Eftekharian F: The risk of hearing loss associated with occupational exposure to organic solvents mixture with and without concurrent noise exposure: A systematic review and meta-analysis. International journal of occupational medicine and environmental health 2017, 30(4):521-535.

23.Weiqun X, Meixiang K, Jun X, Yifei Z, Jieying Z, Wei Y: Investigation on a case report of occupational acute trichloromethane poisoning. China Occupational Medicine 2014, 41(1):119-120.

24.Lionte C: Lethal complications after poisoning with chloroform-case report and literature review. Human \& Experimental Toxicology 2010, 29(7):615622.

25.Jinxia C: Investigation on a case report of occupational acute trichloromethane poisoning. Occupational Health and Emergency Rescue $2012,30(2): 101$.

26. Huang Hutao, Zhang Ping, Li Keyong, Wang Jie, Zheng Guang, Xuetao. Z: Advances in research on health damage caused by dimethylformamide. Occupational Health and Emergency Rescue 2018, 36(05):398-401+441.

\section{Tables}

Table 1. Annual change trends for new case of OSODs in Guangdong Province, 2006ロ2015, n(\%)

\begin{tabular}{|c|c|c|c|c|c|c|c|c|c|c|c|}
\hline OSODs & 2006 & 2007 & 2008 & 2009 & 2010 & 2011 & 2012 & 2013 & 2014 & 2015 & Total \\
\hline Benzolism & $38(6.4)$ & $40(6.8)$ & $26(4.4)$ & $41(6.9)$ & $50(8.4)$ & $65(11.0)$ & $76(12.8)$ & $72(12.2)$ & $121(20.4)$ & $63(10.6)$ & $592(100.0)$ \\
\hline Benzene-induced Leukemia & $2(0.9)$ & $6(2.6)$ & $6(2.6)$ & $12(5.2)$ & $34(14.7)$ & $43(18.5)$ & $36(15.5)$ & 23(9.9) & $39(16.8)$ & $31(13.4)$ & $232(100.0)$ \\
\hline N-hexane Poisoning & $26(12.6)$ & $5(2.4)$ & $32(15.5)$ & $0(0)$ & $44(21.4)$ & $10(4.9)$ & $48(23.3)$ & 11(5.3) & $23(11.2)$ & $7(3.4)$ & $206(100.0)$ \\
\hline $\begin{array}{l}\text { Trichloroethylene-induced } \\
\text { Medicamentosa-like Dermatitis }\end{array}$ & $23(24.5)$ & $5(5.3)$ & $6(6.4)$ & $2(2.1)$ & $9(9.6)$ & $8(8.5)$ & $2(2.1)$ & 12(12.8) & 14(14.9) & 13(13.8) & $94(100.0)$ \\
\hline 1,2-Dichloroethane Poisoning & $0(0)$ & $0(0)$ & $0(0)$ & $5(7.7)$ & 12(18.5) & 12(18.5) & $34(52.3)$ & $1(1.5)$ & $1(1.5)$ & $0(0)$ & $65(100.0)$ \\
\hline Trichloromethane Poisoning & 1(1.9) & $5(9.6)$ & $0(0)$ & $31(59.6)$ & $3(5.8)$ & $2(3.8)$ & $9(17.3)$ & $1(1.9)$ & $0(0)$ & $0(0)$ & $52.0(100.0)$ \\
\hline Dimethyl Formamide Poisoning & $36(73.5)$ & $4(8.2)$ & $0(0)$ & $1(2.0)$ & $1(2.0)$ & $3(6.1)$ & $0(0)$ & $0(0)$ & 2(4.1) & $2(4.1)$ & $49(100.0)$ \\
\hline Total & $126(9.8)$ & $65(5.0)$ & $70(5.4)$ & $92(7.1)$ & $153(11.9)$ & $143(11.1)$ & $205(15.9)$ & $120(9.3)$ & $200(15.5)$ & $116(9.0)$ & $1290(100.0)$ \\
\hline
\end{tabular}

ıble 2-1 Business, ownership type, industry, and enterprise scale, distribution of 7 major OSODs, n(\%)

\begin{tabular}{|c|c|c|c|c|c|c|}
\hline \multirow[t]{2}{*}{ OSODs } & \multirow[t]{2}{*}{ Total } & \multicolumn{5}{|c|}{ ownership type ${ }^{2}$} \\
\hline & & A & B & $\mathrm{C}$ & $\mathrm{D}$ & $\mathrm{E}$ \\
\hline Benzolism & 592 & $200(33.8)$ & $177(29.9)$ & $6(1.0)$ & $29(4.9)$ & $180(30.4)$ \\
\hline Benzene-induced Leukemia & 232 & $45(19.4)$ & $90(38.8)$ & $12(5.2)$ & $14(6.0)$ & $71(30.6)$ \\
\hline N-hexane Poisoning & 206 & $2(1.0)$ & $153(74.3)$ & $4(1.9)$ & $1(0.5)$ & $46(22.3)$ \\
\hline Trichloroethylene-induced Medicamentosa-like Dermatitis & 94 & $6(6.4)$ & $67(71.3)$ & $7(7.4)$ & $1(1.1)$ & $13(13.8)$ \\
\hline 1,2 Dichloroethane Poisoning & 65 & - & $64(98.5)$ & - & - & $1(1.5)$ \\
\hline Trichloromethane Poisoning & 52 & $3(5.8)$ & $47(90.4)$ & - & - & $2(3.8)$ \\
\hline Dimethyl Formamide Poisoning & 49 & $2(4.1)$ & $40(81.6)$ & $1(2.0)$ & - & $6(12.2)$ \\
\hline Total & 1290 & $258(20.0)$ & $638(49.5)$ & $30(2.3)$ & $45(3.5)$ & $319(24.7)$ \\
\hline
\end{tabular}

B, C, D, E represent “Foreign Funded Economy”, " Private Economy”, “Collective Economy”, "State-owned Economy”, ” Hong g/Macao/Taiwan Economy” respectively.

Table 2-2 Business, ownership type, industry, and enterprise scale, distribution of OSODs, n(\%) 


\begin{tabular}{|c|c|c|c|c|c|c|c|c|c|c|c|c|}
\hline \multirow[t]{2}{*}{ OSODs } & \multicolumn{7}{|c|}{ Industry ${ }^{1}$} & \multicolumn{5}{|c|}{ Enterprise scale ${ }^{2}$} \\
\hline & $\mathbf{A}$ & B & $\mathrm{C}$ & $\mathrm{D}$ & $\mathrm{E}$ & $\mathbf{F}$ & G & $\mathrm{a}$ & $\mathbf{b}$ & c & d & e \\
\hline Benzolism & $80(13.5)$ & $75(12.7)$ & $49(8.3)$ & 0 & $21(3.5)$ & $260(43.9)$ & $107(18.1)$ & $126(21.3)$ & $220(37.2)$ & $172(29.1)$ & $2(0.3)$ & $72(12.2)$ \\
\hline Benzene-induced & $33(14.2)$ & $41(17.7)$ & $23(9.9)$ & $2(0.9)$ & $4(1.7)$ & $86(37.1)$ & $43(18.5)$ & $52(22.4)$ & $77(33.2)$ & $81(34.9)$ & $1(0.4)$ & $21(9.1)$ \\
\hline \multicolumn{13}{|l|}{ Leukemia } \\
\hline N-hexane & $122(59.2)$ & $3(1.5)$ & $3(1.5)$ & 0 & 0 & $22(10.7)$ & $56(27.2)$ & 0 & $44(21.4)$ & $137(66.5)$ & $1(0.5)$ & $24(11.7)$ \\
\hline \multicolumn{13}{|l|}{ Poisoning } \\
\hline Trichloroethylene- & $46(48.9)$ & 18(19.1) & 0 & 0 & 0 & $10(10.6)$ & $20(21.3)$ & $8(8.5)$ & $19(20.2)$ & $43(45.7)$ & $1(1.1)$ & $23(24.5)$ \\
\hline \multicolumn{13}{|l|}{ induced } \\
\hline \multicolumn{13}{|l|}{ Medicamentosa- } \\
\hline \multicolumn{13}{|l|}{ like Dermatitis } \\
\hline 1,2-Dichloroethane & 0 & $4(6.2)$ & $2(3.1)$ & 0 & 0 & $56(86.2)$ & $3(4.6)$ & 0 & $4(6.2)$ & $60(92.3)$ & 0 & $1(1.5)$ \\
\hline \multicolumn{13}{|l|}{ Poisoning } \\
\hline Trichloromethane & $40(76.9)$ & 0 & $1(1.9)$ & 0 & 0 & $7(13.5)$ & $4(7.7)$ & $30(57.7)$ & $3(5.8)$ & $6(11.5)$ & 0 & $13(25.0)$ \\
\hline \multicolumn{13}{|l|}{ Poisoning } \\
\hline Dimethyl & 0 & 0 & 0 & 0 & 0 & $47(95.9)$ & $2(4.1)$ & 0 & 7(14.3) & 39(79.6) & 0 & $3(6.1)$ \\
\hline \multicolumn{13}{|l|}{ Formamide } \\
\hline \multicolumn{13}{|l|}{ Poisoning } \\
\hline Total & $321(24.9)$ & $141(10.9)$ & $78(6.0)$ & $2(.02)$ & $25(1.9)$ & 488(37.8) & $235(18.2)$ & 216(16.7) & $374(29.0)$ & $538(41.7)$ & $5(0.4)$ & $157(12.2)$ \\
\hline
\end{tabular}

${ }^{1}$ A, B , C, D, E, F, G represent "Electronic Industry", "Machinery industry", "Building Materials Industry", "Construction Industry", “Mineral Industry” ,"Light Industry” , “Others”respectively. ${ }^{2}$ a, b, c, d, e represent "Large-scale”, "Middle-scale”, "Small-scale”, "Microscale", "Unknown" respectively.

Table 3. Annual change trends of onset age of OSODs in Guangdong Province, 2006ロ2015, $M\left(\mathrm{P}_{25 \square} \mathrm{P}_{75}\right)$ *

\begin{tabular}{|c|c|c|c|c|c|c|c|c|c|c|c|}
\hline OSODs & 2006 & 2007 & 2008 & 2009 & 2010 & 2011 & 2012 & 2013 & 2014 & 2015 & Total \\
\hline Benzolism & 32.5 & 31 & 34.5 & 34 & 32.5 & 38 & 37 & 38 & 40 & 42 & 37 \\
\hline Benzene-induced Leukemia & $\begin{array}{c}(29,37) \\
33.5\end{array}$ & $\begin{array}{l}(22.5,39.5) \\
30\end{array}$ & $\begin{array}{l}(30,40) \\
35\end{array}$ & $\begin{array}{c}(30,39) \\
27.5\end{array}$ & $\begin{array}{c}(26,37) \\
34.5\end{array}$ & $\begin{array}{c}(32,42) \\
37\end{array}$ & $\begin{array}{c}(30.5,41) \\
36.5\end{array}$ & $\begin{array}{c}(31,42) \\
38\end{array}$ & $\begin{array}{l}(34,45) \\
35\end{array}$ & $\begin{array}{c}(37,46) \\
36\end{array}$ & $\begin{array}{l}(31,42) \\
35\end{array}$ \\
\hline N-hexane Poisoning & $\begin{array}{c}(33,34) \\
23\end{array}$ & $\begin{array}{c}(22,33) \\
35\end{array}$ & $\begin{array}{c}(32,39) \\
25\end{array}$ & $(23,32)$ & $\begin{array}{c}(28,39) \\
21\end{array}$ & $\begin{array}{c}(29,42) \\
20\end{array}$ & $\begin{array}{c}(31,43.5) \\
26.5\end{array}$ & $\begin{array}{c}(32,43) \\
23\end{array}$ & $\begin{array}{c}(28,41) \\
27\end{array}$ & $\begin{array}{c}(30,45) \\
33\end{array}$ & $\begin{array}{c}(29,42) \\
23\end{array}$ \\
\hline Trichloroethylene-induced & $\begin{array}{c}(20,27) \\
25\end{array}$ & $\begin{array}{c}(33,37) \\
27\end{array}$ & $\begin{array}{l}(21,28) \\
22.5\end{array}$ & 28.5 & $\begin{array}{l}(19.5,23.5) \\
22\end{array}$ & $\begin{array}{c}(17,22) \\
25\end{array}$ & $\begin{array}{l}(19,37.5) \\
25.5\end{array}$ & $\begin{array}{c}(20,26) \\
31.5\end{array}$ & $\begin{array}{c}(20,32) \\
25\end{array}$ & $\begin{array}{l}(30,45) \\
29\end{array}$ & $\begin{array}{c}(20,28) \\
26\end{array}$ \\
\hline 1,2-Dichloroethane Poisoning & $(21,33)$ & $(25,35)$ & $(18,27)$ & $\begin{array}{c}(28,29) \\
32\end{array}$ & $\begin{array}{l}(21,31) \\
28.5\end{array}$ & $\begin{array}{c}(20,27.5) \\
29\end{array}$ & $\begin{array}{c}(24,27) \\
23.5\end{array}$ & $\begin{array}{c}(25,40.5) \\
51\end{array}$ & $\begin{array}{c}(22,31) \\
21\end{array}$ & $(21,37)$ & $\begin{array}{c}(22,33) \\
26\end{array}$ \\
\hline Trichloromethane Poisoning & 18 & 29 & - & $\begin{array}{c}(26,36) \\
19\end{array}$ & $\begin{array}{c}(23,32) \\
32\end{array}$ & $\begin{array}{c}(22.5,40.5) \\
28\end{array}$ & $\begin{array}{c}(17,38) \\
35\end{array}$ & $\begin{array}{c}(51,51) \\
42\end{array}$ & $\begin{array}{c}(21,21) \\
-\end{array}$ & - & $\begin{array}{c}(20,38) \\
21\end{array}$ \\
\hline Dimethyl Formamide Poisoning & $\begin{array}{c}(18,18) \\
24\end{array}$ & $\begin{array}{c}(23,30) \\
23.5\end{array}$ & - & $\begin{array}{c}(18,21) \\
28\end{array}$ & $\begin{array}{c}(28,42) \\
32\end{array}$ & $\begin{array}{c}(20,36) \\
38\end{array}$ & $\begin{array}{c}(25,44) \\
-\end{array}$ & $(42,42)$ & 52 & 37.5 & $\begin{array}{c}(18,29) \\
28\end{array}$ \\
\hline & $(20,30)$ & $(21,26.5)$ & & $(28,28)$ & $(32,32)$ & $(28,39)$ & & & $(49,55)$ & $(37,38)$ & $(21,33)$ \\
\hline
\end{tabular}

${ }^{*}$ M: Median; $\left(\mathrm{P}_{25}, \mathrm{P}_{75}\right)$ : Range Interquartile.

Table 4. Annual change trend of working age of onset OSODs in Guangdong Province, 2006ロ2015, M( $\left.P_{25} \square P_{75}\right) *$ 


\begin{tabular}{|c|c|c|c|c|c|c|c|c|c|c|c|}
\hline OSODS & 2006 & 2007 & 2008 & 2009 & 2010 & 2011 & 2012 & 2013 & 2014 & 2015 & Total \\
\hline Benzolism(Year) & $4(2,5)$ & $3(1,5)$ & $6(3,10)$ & $3(1,4)$ & $2(1,4)$ & $4(2,7)$ & $5(3,9)$ & $6(2,9)$ & $6(2,10)$ & $7(4,10)$ & $4(2,8)$ \\
\hline Benzene-induced Leukemia(Year) & $3.5(2,5)$ & $1.5(1,3)$ & $9(4,12)$ & $2.5(1,4)$ & $4(2,6)$ & $4(2,9)$ & $4(2.5,8)$ & $6(3,8)$ & $4(2,7)$ & $8(4,10)$ & $4(2,8)$ \\
\hline N-hexane Poisoning(Month) & $9(7,12)$ & $56(11,108)$ & $8(5,18)$ & $7(5,10)$ & $9(6,12)$ & $12(7,15)$ & $6(6,6)$ & $8(6,6)$ & $8(6,12)$ & $10(9,14)$ & $8(6,13)$ \\
\hline $\begin{array}{l}\text { Trichloroethylene-induced } \\
\text { Medicamentosa-like Dermatitis(Month) }\end{array}$ & $1(1,1)$ & $1(1,2)$ & $1(1,1)$ & 17(1,32) & $1(1,1)$ & $1(1,2)$ & $2(1,2)$ & $1(1,1)$ & $1(1,1)$ & $1(0,1)$ & $1(1,1)$ \\
\hline Dichloroethan & - & & - & 3( & $6(2$ & 2( & $7(4$ & 1 & & - & $5(3,10)$ \\
\hline Trichloromethan & 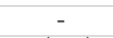 & $6(3,7)$ & - & $1(1,1)$ & $2(1,4)$ & $32(4,60)$ & $1(1,37)$ & $1(1,1)$ & $29(29,29)$ & I & $1(1,4)$ \\
\hline Dimethyl Formamide Poisoning(Month) & $1(1,1)$ & $2(1,3)$ & - & $5(5,5)$ & $54(54,54)$ & $8(0,10)$ & & & $1(1,1)$ & $46(2,90)$ & $1(1,1)$ \\
\hline
\end{tabular}

${ }^{*} \mathrm{M}$ : Median; $\left(\mathrm{P}_{25}, \mathrm{P}_{75}\right)$ : Range Interquartile.

\section{Figures}

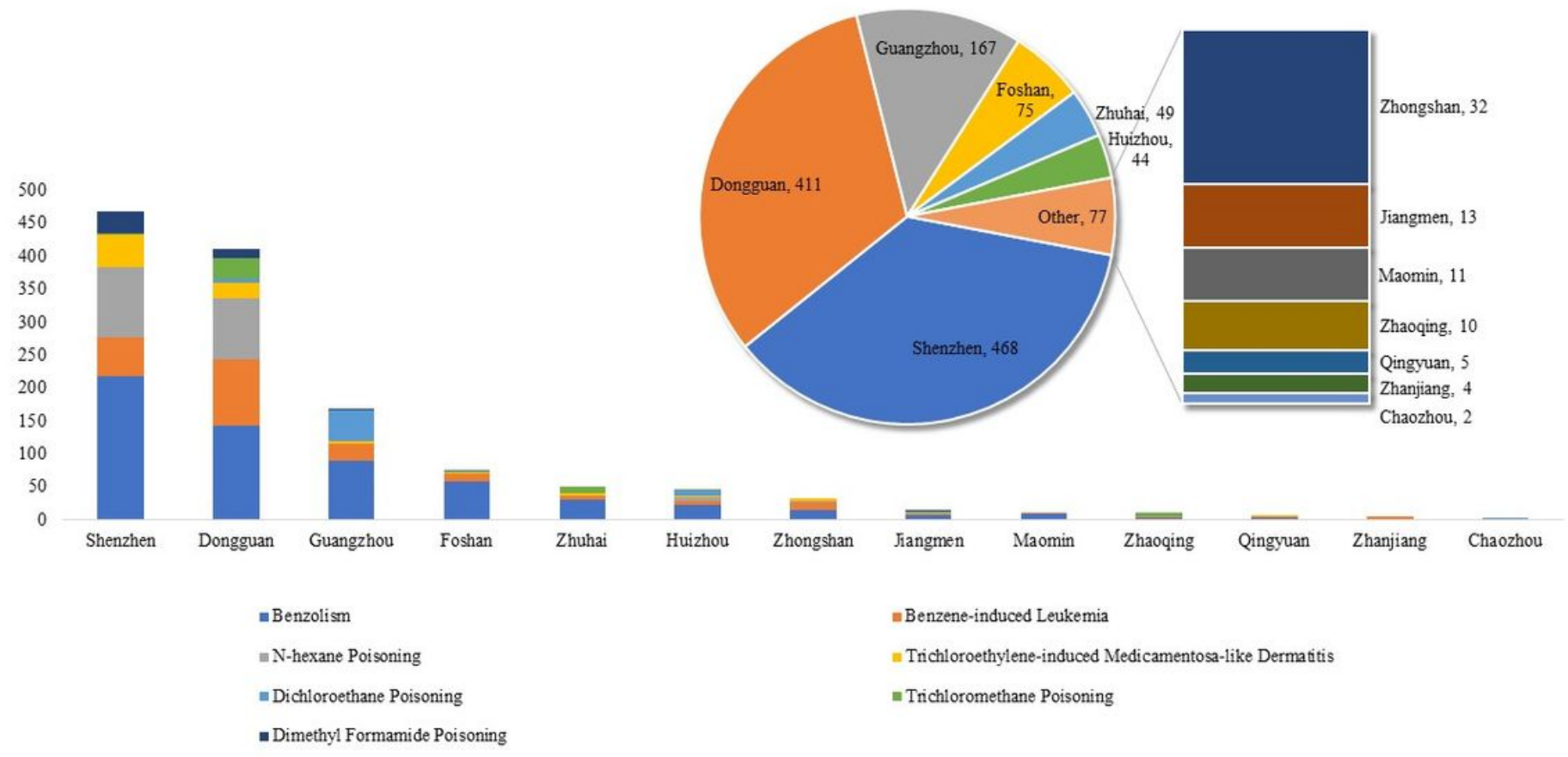

\section{Figure 1}

Districts of 7 Major OSODs in Guangdong Province, China, 2006-2015, n(\%) 


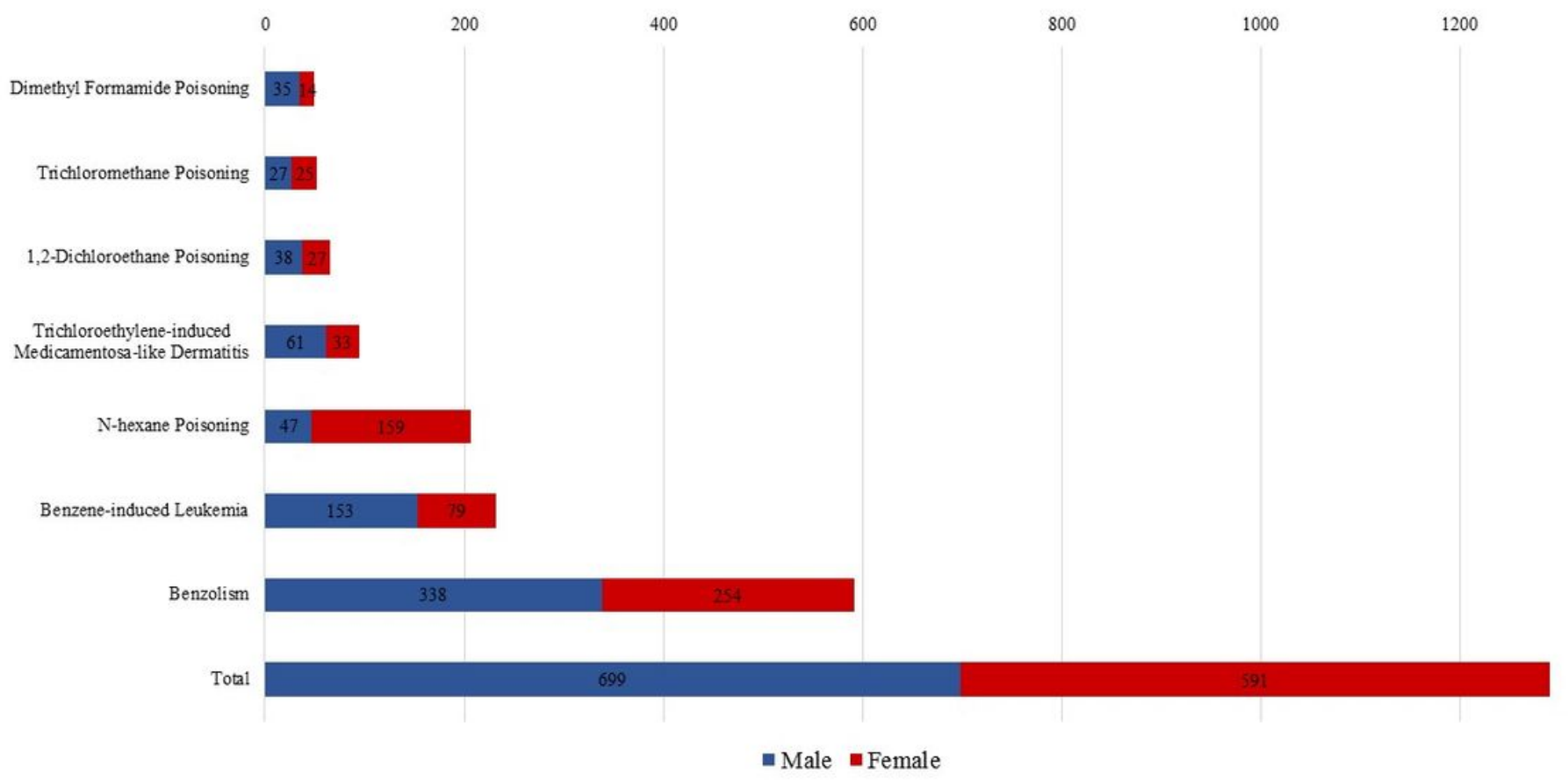

Figure 2

Gender Distribution of 7 Major OSODs in Guangdong Province, China, 2006-2015 